\title{
Effects of the COVID-19 Pandemic on Routine Pediatric Vaccine Ordering and Administration — United States, 2020
}

\begin{abstract}
Jeanne M. Santoli, MD ${ }^{1}$; Megan C. Lindley, MPH${ }^{1}$; Malini B. DeSilva, MD²; Elyse O. Kharbanda, MD²; Matthew F. Daley, MD 3 ; Lisa Galloway ${ }^{1}$; Julianne Gee, $\mathrm{MPH}^{4}$; Mick Glover ${ }^{5}$; Ben Herring ${ }^{6}$; Yoonjae Kang, $\mathrm{MPH}^{1}$; Paul Lucas, MS ${ }^{1}$; Cameron Noblit, MPH${ }^{1}$; Jeanne Tropper, MPH, MS, MBA ${ }^{1}$; Tara Vogt, $\mathrm{PhD}^{1}$; Eric Weintraub, $\mathrm{MPH}^{4}$
\end{abstract}

On May 8, 2020, this report was posted as an MMWR Early Release on the MMWR website (https://www.cdc.gov/mmwr).

On March 13, 2020, the president of the United States declared a national emergency in response to the coronavirus disease 2019 (COVID-19) pandemic (1). With reports of laboratory-confirmed cases in all 50 states by that time (2), disruptions were anticipated in the U.S. health care system's ability to continue providing routine preventive and other nonemergency care. In addition, many states and localities issued shelter-in-place or stay-at-home orders to reduce the spread of COVID-19, limiting movement outside the home to essential activities (3). On March 24, CDC posted guidance emphasizing the importance of routine well child care and immunization, particularly for children aged $\leq 24$ months, when many childhood vaccines are recommended.*

Two data sources were examined to assess the impact of the pandemic on pediatric vaccination in the United States: Vaccines for Children Program (VFC) provider order data from CDC's Vaccine Tracking System and Vaccine Safety Datalink (VSD) vaccine administration data. Vaccination coverage is the traditional metric used to assess vaccine usage; however, provider orders and doses administered represent two immediately available proxy measures.

VFC is a national program that provides federally purchased vaccines to approximately $50 \%$ of U.S. children aged 0-18 years. ${ }^{\dagger}$ Cumulative doses of VFC-funded vaccines ordered by health care providers at weekly intervals during two periods (January 7, 2019-April 21, 2019 [period 1] and January 6, 2020-April 19, 2020 [period 2]) were tallied, and differences in cumulative weekly vaccine doses ordered between period 2 and period 1 were calculated for all noninfluenza vaccines $\$$ that the Advisory Committee on Immunization Practices (ACIP) recommends for children and, as an example,

\footnotetext{
*https://www.cdc.gov/coronavirus/2019-ncov/hcp/pediatric-hcp.html.

$\dagger$ Children aged $\leq 18$ years are eligible if they are Medicaid-eligible, uninsured,

American Indian/Alaska Native, or underinsured and vaccinated at federally qualified health centers, rural health clinics, or provider sites with an approved deputization agreement with the state public health department. https://www. cdc.gov/vaccines/programs/vfc/index.html.

${ }^{\S}$ https://www.cdc.gov/vaccines/schedules/hcp/imz/child-adolescent.html.
}

for measles-containing-vaccines. VSD is a collaborative project between CDC's Immunization Safety Office and eight U.S. health care organizations serving publicly and privately insured patients. ${ }^{* *}$ Aggregate counts of measles-containing vaccine doses administered each week at VSD sites during period 2 were compared between two pediatric age groups: children aged $\leq 24$ months and those aged $>24$ months through 18 years.

Vaccine Tracking System data indicate a notable decrease in orders for VFC-funded, ACIP-recommended noninfluenza childhood vaccines and for measles-containing vaccines during period 2 compared with period 1 (Figure). The decline began the week after the national emergency declaration; similar declines in orders for other vaccines were also observed. VSD data show a corresponding decline in measles-containing vaccine administrations beginning the week of March 16, 2020. The decrease was less prominent among children aged $\leq 24$ months than among older children (Figure). The subsequent increase in vaccine administrations observed in late March was more prominent in younger than older children.

The substantial reduction in VFC-funded pediatric vaccine ordering after the COVID-19 emergency declaration is consistent with changes in vaccine administration among children in the VSD population receiving care through eight large U.S. health care organizations. The smaller decline in measles-containing vaccine administration among children aged $\leq 24$ months suggests that system-level strategies to prioritize well child care and immunization for this age group are being implemented. Increases in vaccine administration to children aged $\leq 24$ months beginning in late March might reflect early success of strategies implemented by VSD health care organizations to promote childhood vaccinations in the context of the pandemic, including outreach to patients overdue for vaccinations and changing office workflows to minimize contact between patients (4). Assessment of state and local

\footnotetext{
In the United States, two measles-containing vaccines are licensed for routine use in children: measles-mumps-rubella (MMR) vaccine and a combination MMR and varicella vaccine (MMRV). The Advisory Committee on Immunization Practices recommends that U.S. children receive a 2-dose series of measles-containing vaccines at ages $12-15$ months and $4-6$ years. ** https://www.cdc.gov/vaccinesafety/ensuringsafety/monitoring/vsd/.
} 
FIGURE. Weekly changes in Vaccines for Children Program (VFC) provider orders* and Vaccine Safety Datalink (VSD) doses administered ${ }^{\dagger}$ for routine pediatric vaccines - United States, January 6-April 19, 2020

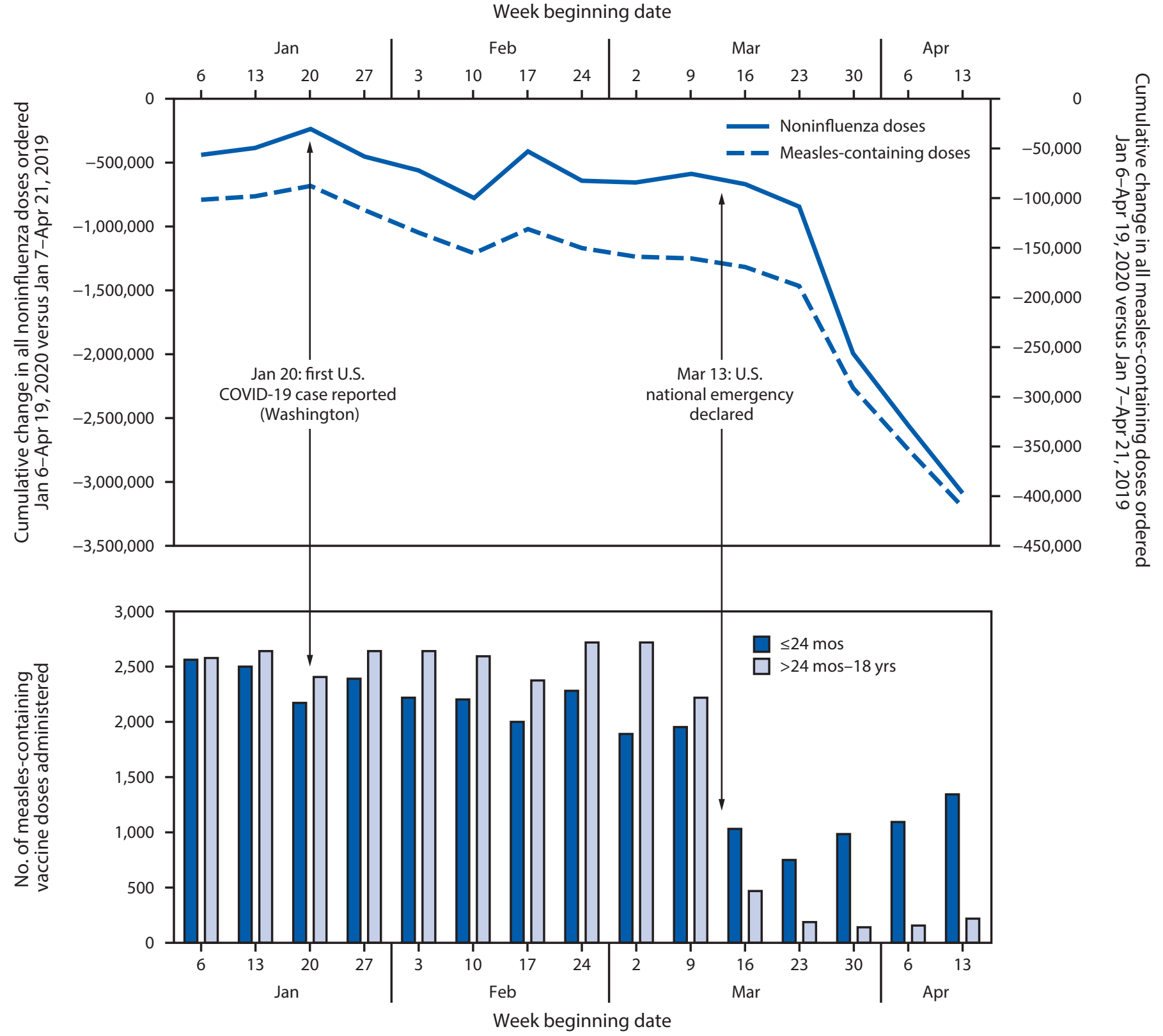

* VFC data represent the difference in cumulative doses of VFC-funded noninfluenza and measles-containing vaccines ordered by health care providers at weekly intervals between Jan 7-Apr 21, 2019, and Jan 6-Apr 19, 2020.

+ VSD data depict weekly measles-containing vaccine doses administered by age group (age $\leq 24$ mos and >24 mos-18 yrs).

vaccination coverage is needed to quantify the impact among U.S. children of all ages and prioritize areas for intervention.

The ongoing COVID-19 pandemic is a reminder of the importance of vaccination. The identified declines in routine pediatric vaccine ordering and doses administered might indicate that U.S. children and their communities face increased risks for outbreaks of vaccine-preventable diseases. Parental concerns about potentially exposing their children to
COVID-19 during well child visits might contribute to the declines observed (5). To the extent that this is the case, reminding parents of the vital need to protect their children against serious vaccine-preventable diseases, even as the COVID-19 pandemic continues, is critical. As social distancing requirements are relaxed, children who are not protected by vaccines will be more vulnerable to diseases such as measles. In response, continued coordinated efforts between health care providers 
and public health officials at the local, state, and federal levels will be necessary to achieve rapid catch-up vaccination.

\section{Acknowledgments}

Vaccine Safety Datalink; Leslie Kuckler, Jingyi Zhu, HealthPartners Institute, Minneapolis, Minnesota.

Corresponding author: Jeanne Santoli, jsantoli@cdc.gov, 404-639-8877.

${ }^{1}$ Immunization Services Division, National Center for Immunization and Respiratory Diseases, CDC; ${ }^{2}$ HealthPartners Institute, Minneapolis, Minnesota; ${ }^{3}$ Institute for Health Research, Kaiser Permanente Colorado, Aurora, Colorado; ${ }^{4}$ Division of Healthcare Quality Promotion, National Center for Emerging Zoonotic and Infectious Diseases, CDC; ${ }^{5}$ Total Solutions, Inc., Madison, Alabama; ${ }^{6}$ Carter Consulting, Inc., Atlanta, Georgia.

All authors have completed and submitted the International Committee of Medical Journal Editors form for disclosure of potential conflicts of interest. No potential conflicts of interest were disclosed.

\section{References}

1. Federal Emergency Management Agency. Bringing resources to state, local, tribal \& territorial governments. Washington, DC: US Department of the Homeland Security, Federal Emergency Management Agency; 2020. https://www.fema.gov/coronavirus/governments

2. Bialek S, Bowen V, Chow N, et al.; CDC COVID-19 Response Team. Geographic differences in COVID-19 cases, deaths, and incidenceUnited States, February 12-April 7, 2020. MMWR Morb Mortal Wkly Rep 2020;69:465-71. https://doi.org/10.15585/mmwr.mm6915e4

3. Financial Industry Regulatory Authority. State "shelter-in-place" and "stay-at-home" orders. Washington, DC: Financial Industry Regulatory Authority; 2020. https://www.finra.org/rules-guidance/key-topics/covid19/shelter-in-place

4. Dunn J. Your kids still need their vaccines, even during coronavirus crisis. Seattle, WA: Seattle'sChild; 2020. http://www.seattleschild.com/ Your-kids-still-need-their-vaccines-even-during-coronavirus-crisis/

5. Hoffman J. Vaccine rates drop dangerously as parents avoid doctor's visits. New York Times. April 23, 2020. https://www.nytimes.com/2020/04/23/ health/coronavirus-measles-vaccines.html 